\title{
ON SECOND VARIATION OF PERELMAN'S RICCI SHRINKER ENTROPY
}

\author{
HUAI-DONG CAO AND MENG ZHU
}

\begin{abstract}
In this paper we provide a detailed proof of the second variation formula, essentially due to Richard Hamilton, Tom Ilmanen and the first author, for Perelman's $\nu$-entropy. In particular, we correct an error in the stability operator stated in Theorem 6.3 of [2]. Moreover, we obtain a necessary condition for linearly stable shrinkers in terms of the least eigenvalue and its multiplicity of certain Lichnerowicz type operator associated to the second variation.
\end{abstract}

\section{The Results}

A complete Riemannian metric $g_{i j}$ on a smooth manifold $M^{n}$ is called a gradient shrinking Ricci soliton if there exists a smooth function $f$ on $M^{n}$ such that the Ricci tensor $R_{i j}$ of the metric $g_{i j}$ satisfies the equation

$$
R_{i j}+\nabla_{i} \nabla_{j} f=\frac{1}{2 \tau} g_{i j}
$$

for some constant $\tau>0$. The function $f$ is called a potential function of the Ricci soliton. When $f$ is a constant we obtain an Einstein metric of positive scalar curvature. Thus, Ricci solitons include Einstein metrics as a special case.

Ricci solitons correspond to self-similar solutions of Hamilton's Ricci flow, and often arise as limits of dilations of singularities in the Ricci flow. In particular shrinking solitons are possible Type I singularity models in the Ricci flow. We refer the readers to [2], 3] and the references therein for more information on Ricci solitons.

Ricci solitons can be viewed as fixed points of the Ricci flow, as a dynamical system, on the space of Riemannian metrics modulo diffeomorphisms and scalings. In [15, Perelman introduced the $\mathcal{W}$-functional

$$
\mathcal{W}\left(g_{i j}, f, \tau\right)=\int_{M}\left[\tau\left(R+|\nabla f|^{2}\right)+f-n\right](4 \pi \tau)^{-\frac{n}{2}} e^{-f} d V
$$

on a compact manifold $M^{n}$, where $g_{i j}$ is a Riemannian metric, $\mathrm{R}$ the scalar curvature, $f$ a smooth function on $M^{n}$, and $\tau$ a positive scale parameter. The associated $\nu$-entropy is defined by

$$
\nu\left(g_{i j}\right)=\inf \left\{\mathcal{W}(g, f, \tau): f \in C^{\infty}(M), \tau>0,(4 \pi \tau)^{-\frac{n}{2}} \int e^{-f} d V=1\right\} .
$$

\footnotetext{
${ }^{1}$ The first author was partially supported by NSF Grants DMS-0506084 and DMS-0909581; the second author was partially supported by NSF Grant DMS-0354621 and a Graduate Summer Fellowship awarded by the School of Arts and Sciences at Lehigh University.
} 
It turns out that the $\nu$-entropy is monotone increasing under the Ricci flow, and its critical points are precisely given by gradient shrinking solitons. In particular, it follows that all compact shrinking Ricci solitons are gradient shrinking solitons, a fact shown by Perelman [15.

In dimensions 2 and 3, Hamilton [11] and Ivey [12] respectively showed that the only compact shrinking solitons are quotients of the round spheres. However, for dimension $n \geq 4$, compact non-Einstein shrinking solitons do exist. Specifically in dimension $n=4$, Koiso [13] and the first author [1] independently constructed a gradient Kähler-Ricci shrinking soliton on $\mathbb{C} P^{2} \#\left(-\mathbb{C} P^{2}\right)$, and Wang-Zhu [16] on $\mathbb{C} P^{2} \#\left(-2 \mathbb{C} P^{2}\right)$, while in the noncompact case Feldman-Ilmanen-Knopf [8] constructed the $U(2)$-invariant gradient shrinking Kähler-Ricci solitons on the tautological line bundle $\mathcal{O}(-1)$ of $\mathbb{C} P^{1}$, the blow-up of $\mathbb{C}^{2}$ at the origin. These are the only known examples of nontrivial (i.e., non-Einstein or non-product) complete shrinking Ricci solitons in dimension 4 so far.

In 4, Hamilton, Ilmanen and the first author initiated the study of linear stability of Ricci solitons. They found the second variation formula of the $\nu$-entropy for positive Einstein manifolds and investigated the linear stability of certain Einstein manifolds. By definition, a Ricci shrinker or Einstein manifold is called linearly stable if the second variation is non-positive. They showed that, while the round sphere $\mathbb{S}^{n}$ and the complex projective space $\mathbb{C} P^{n}$ are linearly stable, many known Einstein manifolds are unstable for the Ricci flow so that generic perturbations acquire higher $\nu$-entropy and thus can never return near the original metric. In particular, all Kähler-Einstein manifolds with Hodge number $h^{1,1}>1$ are unstable.

In dimension $n \geq 4$, so far no one knows how to classify Einstein manifolds of positive scalar curvature, let alone gradient shrinking Ricci solitons. However, as far as applications of the Ricci flow to topology is concerned, one is more interested in stable shrinking solitons since unstable ones could be perturbed away thus may not represent generic singularities. For this reason, it is desirable and important to classify stable shrinking Ricci solitons. Note that, the work of Cao-HamiltonIlmanen 4 suggests that most gradient shrinking Ricci solitons are unstable. In fact, Hamilton conjectured that, at least in dimension $n=4$, compact linearly stable shrinkers are rank one symmetric spaces, namely either the round sphere $\mathbb{S}^{4}$ or the complex projective space $\mathbb{C} P^{2}$ with the Fubini-Study metric. Of course, in studying linear stability of shrinkers, the second variation formula of the $\nu$-entropy is indispensable. In this paper, we present a detailed proof of the second variation formula, first due to Hamilton, Ilmanen and the first author (cf. Theorem 6.3 in [2]), for Ricci shrinkers.

To state the second variation formula, we need some notations first. For any symmetric 2-tensor $h=h_{i j}$ and 1-form $\omega=\omega_{i}$, we denote

$$
\begin{gathered}
R m(h, \cdot):=R_{i j k l} h_{j l}, \\
\operatorname{div} \omega:=\nabla_{i} \omega_{i}, \quad(\operatorname{div} h)_{i}:=\nabla_{j} h_{j i} .
\end{gathered}
$$

Moreover, as done in 2], we define

$$
\operatorname{div}_{f} \omega:=e^{f} \operatorname{div}\left(e^{-f} \omega\right)=\nabla_{i} \omega_{i}-\omega_{i} \nabla_{i} f
$$

and

$$
\operatorname{div}_{f} h:=e^{f} \operatorname{div}\left(e^{-f} h\right)=\operatorname{div} h-h(\nabla f, \cdot),
$$


i.e.,

$$
\left(\operatorname{div}_{f} h\right)_{i}=\nabla_{j} h_{i j}-h_{i j} \nabla_{j} f .
$$

We also define $\operatorname{div}_{f}^{\dagger}$ on 1-forms (and similarly on functions) by

so that

$$
\left(\operatorname{div}_{f}^{\dagger} \omega\right)_{i j}=-\left(\nabla_{i} \omega_{j}+\nabla_{j} \omega_{i}\right) / 2=-(1 / 2) L_{\omega \#} g_{i j}
$$

$$
\int_{M} e^{-f}<\operatorname{div}_{f}^{\dagger} \omega, h>d V=\int_{M} e^{-f}<\omega, \operatorname{div}_{f} h>d V .
$$

Here $\omega^{\#}$ is the vector field dual to $\omega$. Clearly, $\operatorname{div}_{f}^{\dagger}$ is just the adjoint of $\operatorname{div}_{f}$ with respect to the weighted $L^{2}$-inner product

$$
(\cdot, \cdot)_{f}=\int_{M}<\cdot, \cdot>e^{-f} d V
$$

Finally we denote

$$
\Delta_{f}:=\Delta-\nabla f \cdot \nabla
$$

Remark 1.1. If we denote by div* the adjoint of div with respect to the $L^{2}$-inner product

$$
(\cdot, \cdot)=\int_{M}<\cdot, \cdot>d V
$$

then, as pointed out in 2], one can easily verify that

$$
\operatorname{div}_{f}^{\dagger}=\operatorname{div}^{*} .
$$

Now we can state the full second variation formula for Ricci shrinkers:

Theorem 1.1. (Cao-Hamilton-Ilmanen) Let $\left(M^{n}, g_{i j}, f\right)$ be a compact Ricci shrinker with the potential function $f$ and satisfying the Ricci soliton equation (1.1). For any symmetric 2-tensor $h=h_{i j}$, consider variations $g_{i j}(s)=g_{i j}+s h_{i j}$. Then the second variation $\delta_{g}^{2} \nu(h, h)$ is given by

$$
\left.\frac{d^{2}}{d s^{2}}\right|_{s=0} \nu(g(s))=\frac{\tau}{(4 \pi \tau)^{n / 2}} \int_{M}<\hat{N} h, h>e^{-f} d V
$$

where the stability operator $\hat{N}$ is given by

$$
\hat{N} h:=\frac{1}{2} \Delta_{f} h+R m(h, \cdot)+\operatorname{div}_{f}^{\dagger} \operatorname{div}_{f} h+\frac{1}{2} \nabla^{2} \hat{v}_{h}-R c \frac{\int_{M}<R c, h>e^{-f}}{\int_{M} R e^{-f}},
$$

and $\hat{v}_{h}$ is the unique solution of

$$
\Delta_{f} \hat{v}_{h}+\frac{\hat{v}_{h}}{2 \tau}=\operatorname{div}_{f} \operatorname{div}_{f} h, \quad \int_{M} \hat{v}_{h} e^{-f}=0 .
$$

Remark 1.2. As we pointed out before, Theorem 1.1 is essentially due to Hamilton, Ilmanen and the first author (cf. Theorem 6.3 in [2]). However, the coefficient of the last term of the stability operator $\hat{N}$ (which depends on $\delta \tau$, the first variation of the parameter $\tau$ ) was stated incorrectly in [2]. One of our contributions in this paper is to derive an explicit formula for $\delta \tau$ (see Lemma 2.4 below), thus obtaining the correct coefficient and hence a complete second variation formula for Ricci shrinkers. Of course, it would be interesting to investigate the noncompact case as well. In this case, the asymptotic estimates on potential functions and volume 
growth upper bound proved by Cao-Zhou [5], and an integral bound on the Ricci curvature by Munteanu-Sesum [14] should be very helpful. We point out that, while the stability operator $\hat{N}$ is already quite useful even without knowing the explicit coefficient of the last term, it will be rather crucial to have this explicit and correct coefficient in efforts of trying to classify stable shrinkers. For example, this explicit coefficient is essential in showing that the Ricci tensor is a null eigen-tensor of $\hat{N}$ (see Lemma 3.3) which rules out any hope of using the Ricci tensor as a possible unstable direction.

Remark 1.3. In the very recent work [10, Stuart Hall and Thomas Murphy proved that Kähler-Ricci shrinking solitons with Hodge number $h^{1,1}>1$ are unstable, thus extending the results of Cao-Hamilton-Ilmanen [4] in the Kähler-Einstein case mentioned above. In the course of their proof, they also verified the second variation formula stated in 2, though didn't find out explicitly the coefficient of the last term of $\hat{N}$ (which does not affect the proof of their result since they only considered certain special variations orthogonal to $R c$ ).

Remark 1.4. If $\left(M^{n}, g_{i j}\right)$ is Einstein with $R c=\frac{1}{2 \tau} g_{i j}$, Theorem 1.1 reduces to

Theorem 1.2. (Cao-Hamilton-Ilmanen [4) Let $\left(M^{n}, g_{i j}\right)$ be a Einstein manifold and consider variations $g_{i j}(s)=g_{i j}+s h_{i j}$. Then the second variation $\delta_{g}^{2} \nu(h, h)$ is given by

where

$$
\left.\frac{d^{2}}{d s^{2}}\right|_{s=0} \nu(g(s))=\frac{\tau}{\operatorname{Vol}(M, g)} \int_{M}<N h, h>d V
$$

$$
N h:=\frac{1}{2} \Delta h+R m(h, \cdot)+\operatorname{div}^{*} \operatorname{div} h+\frac{1}{2} \nabla^{2} v_{h}-\frac{g}{2 n \tau \operatorname{Vol}(M, g)} \int_{M} \operatorname{tr}_{g} h d V,
$$

and $v_{h}$ is the unique solution of

$$
\Delta v_{h}+\frac{v_{h}}{2 \tau}=\operatorname{div} \operatorname{div} h, \quad \int_{M} v_{h}=0 .
$$

Finally, using the second variation formula, we obtain the following necessary condition for linearly stable shrinkers:

Theorem 1.3. Suppose $\left(M^{n}, g_{i j}, f\right)$ is a compact linearly stable shrinking soliton satisfying (1.1), then $-\frac{1}{2 \tau}$ is the only negative eigenvalue of the operator $\mathcal{L}_{f}$ (with Rc being an eigen-tensor), defined by

$$
\mathcal{L}_{f} h=\frac{1}{2} \Delta h+R m(h, \cdot),
$$

on $\operatorname{ker}_{\operatorname{div}_{f}}$ and the multiplicity of $-\frac{1}{2 \tau}$ is one. In particular, $-\frac{1}{2 \tau}$ is the least eigenvalue of $\mathcal{L}_{f}$ on $\operatorname{ker}_{\operatorname{div}_{f}}$.

Remark 1.5. In proving Theorem 1.3, the explicit coefficient of Rc term in $\hat{N}$ is not needed.

Remark 1.6. In the mean curvature flow, Colding and Minicozzi [7] have shown that for any shrinker its mean curvature $H$ is an eigenfunction of certain operator involved in the corresponding stability operator, and that for any (linearly) stable shrinker the mean curvature function $H$ belongs to the least eigenvalue of the operator which in turn implies that $H$ does not change sign. This fact and a prior 
theorem of Huisken allow them to classify compact stable mean curvature shrinkers. Our Theorem 1.3 above can be considered as the Ricci flow analogy of their results.

Acknowledgements. The first author would like to thank Qiang Chen, Richard Hamilton, Tom Ilmanen for stimulating discussions, and Stuart Hall for helpful communications.

\section{The Proof of Theorem 1.1}

In this section, we describe the first variation of the $\nu$-entropy and derive the second variation formula as stated in Theorem 1.1.

On any given compact manifold $M^{n}$, Perelman [15] introduced the $\mathcal{W}$-functional

$$
\mathcal{W}\left(g_{i j}, f, \tau\right)=\int_{M}\left[\tau\left(R+|\nabla f|^{2}\right)+f-n\right](4 \pi \tau)^{-\frac{n}{2}} e^{-f},
$$

where $g_{i j}$ is a Riemannian metric, $\mathrm{R}$ the scalar curvature, $f$ a smooth function on $M^{n}$, and $\tau$ a positive scale parameter. Clearly the functional $\mathcal{W}$ is invariant under simultaneous scaling of $\tau$ and $g_{i j}$, and invariant under diffeomorphisms. Namely, for any positive number $a$ and any diffeomorphism $\varphi$ we have

$$
\mathcal{W}\left(a \varphi^{*} g_{i j}, \varphi^{*} f, a \tau\right)=\mathcal{W}\left(g_{i j}, f, \tau\right) .
$$

Lemma 2.1. (Perelman [15, see also Lemma 1.5.7 in [6]) If $h_{i j}=\delta g_{i j}, \phi=$ $\delta f$, and $\eta=\delta \tau$, then

$$
\begin{aligned}
& \delta \mathcal{W}\left(h_{i j}, \phi, \eta\right) \\
= & (4 \pi \tau)^{-\frac{n}{2}}\left(\int_{M}-\tau h_{i j}\left(R_{i j}+\nabla_{i} \nabla_{j} f-\frac{1}{2 \tau} g_{i j}\right) e^{-f}\right. \\
& +\int_{M}\left(\frac{1}{2} \operatorname{tr}_{g} h-\phi-\frac{n}{2 \tau} \eta\right)\left[\tau\left(R+2 \Delta f-|\nabla f|^{2}\right)+f-n-1\right] e^{-f} \\
& \left.+\int_{M} \eta\left(R+|\nabla f|^{2}-\frac{n}{2 \tau}\right) e^{-f}\right) .
\end{aligned}
$$

Now, recall that the associated $\nu$-energy is defined by

$$
\nu\left(g_{i j}\right)=\inf \left\{\mathcal{W}(g, f, \tau): f \in C^{\infty}(M), \tau>0\right\},
$$

subject to the constraint

$$
(4 \pi \tau)^{-\frac{n}{2}} \int e^{-f}=1
$$

One checks that $\nu\left(g_{i j}\right)$ is realized by a pair $(f, \tau)$ that solve the equations

$$
\tau\left(-2 \Delta f+|D f|^{2}-R\right)-f+n+\nu=0,
$$

and

$$
(4 \pi \tau)^{-\frac{n}{2}} \int f e^{-f}=\frac{n}{2}+\nu .
$$

For any symmetric 2-tensor $h=h_{i j}$, consider variations $g_{i j}(s)=g_{i j}+s h_{i j}$. Using Lemma 2.1, (2.2) and (2.3), one obtains the following first variation for the $\nu$-entropy.

Lemma 2.2. The first variation $\delta_{g} \nu(h)$ of the $\nu$-entropy is given by

$$
\begin{aligned}
\frac{d}{d s} \nu\left(g_{i j}(s)\right) & =(4 \pi \tau)^{-\frac{n}{2}} \int-\tau<h, R c+\nabla^{2} f-\frac{1}{2 \tau} g>e^{-f} d V \\
& =(4 \pi \tau)^{-\frac{n}{2}} \int-\tau h_{i j}\left(R_{i j}+\nabla_{i} \nabla_{j} f-\frac{1}{2 \tau} g_{i j}\right) e^{-f} d V
\end{aligned}
$$


A stationary point of $\nu$ thus satisfies the Ricci soliton equation (1.1):

$$
R_{i j}+\nabla_{i} \nabla_{j} f-\frac{1}{2 \tau} g_{i j}=0,
$$

which says that $g_{i j}$ is a gradient shrinking Ricci soliton.

Note that, by diffeomorphism invariance of $\nu, \delta_{g} \nu(h)$ vanishes on Lie derivatives, hence on $h_{i j}=\nabla_{i} \nabla_{j} f=\frac{1}{2} L_{\nabla f} g_{i j}$. By scale invariance it also vanishes on multiplies of the metric. Inserting $h_{i j}=-2\left(R_{i j}+\nabla_{i} \nabla_{j} f-\frac{1}{2 \tau} g_{i j}\right)$, one recovers Perelman's formula that finds that $\nu\left(g_{i j}(t)\right)$ is monotone increasing on the Ricci flow, and constant if and only if $g_{i j}(t)$ is a gradient shrinking Ricci soliton. In particular, it follows that any compact shrinking Ricci soliton is necessarily a gradient soliton, a result first shown by Perelman [15].

Now we are going to derive the second variation formula.

Proof of Theorem 1.1. From the first variation formula in Lemma 2.2 we see that the second variation at a gradient shrinker $\left(M^{n}, g_{i j}, f\right)$ is given by

$$
\begin{aligned}
\delta^{2} \nu_{g}(h, h)= & (4 \pi \tau)^{-\frac{n}{2}} \int-\tau<h, \delta\left(R c+\nabla^{2} f-\frac{1}{2 \tau} g\right)>e^{-f} \\
= & (4 \pi \tau)^{-\frac{n}{2}} \int-\tau<h, \delta R c+\delta \nabla^{2} f-\frac{1}{2 \tau} h>e^{-f} \\
& +(4 \pi \tau)^{-\frac{n}{2}}\left(-\frac{\delta \tau}{2 \tau}\right) \int_{M} \operatorname{tr}_{g} h e^{-f} .
\end{aligned}
$$

Lemma 2.3. We have

$$
\delta R c+\delta \nabla^{2} f-\frac{1}{2 \tau} h=-\frac{1}{2} \Delta_{f} h-R m(h, \cdot)-\operatorname{div}_{f}^{\dagger} \operatorname{div}_{f} h-\nabla^{2}\left(-\delta f+\frac{1}{2} \operatorname{tr}_{g} h\right) .
$$

Proof. First of all, it is well-known that the variation $\delta R c$ of the Ricci tensor is given by

$$
(\delta R c)_{i j}=-R_{i k j l} h_{k l}+\frac{1}{2}\left(\nabla_{i} \nabla_{k} h_{j k}+\nabla_{j} \nabla_{k} h_{i k}+R_{i k} h_{j k}+R_{j k} h_{i k}-\Delta h_{i j}-\nabla_{i} \nabla_{j} \operatorname{tr}_{g} h\right),
$$

and, by direct computations (see, e.g., [17]),

$$
\left(\delta \nabla^{2} f\right)_{i j}=\nabla_{i} \nabla_{j}(\delta f)-\frac{1}{2}\left(\nabla_{i} h_{j k}+\nabla_{j} h_{i k}-\nabla_{k} h_{i j}\right) \nabla_{k} f .
$$

On the other hand, by the definition of $\operatorname{div}_{f}$ and $\operatorname{div}_{f}^{\dagger}$ and using the shrinking soliton equation (1.1), we have

$$
\begin{aligned}
\operatorname{div}_{f}^{\dagger} \operatorname{div}_{f} h= & -\frac{1}{2}\left[\nabla_{i}\left(\operatorname{div}_{f} h\right)_{j}+\nabla_{j}\left(\operatorname{div}_{f} h\right)_{i}\right] \\
= & -\frac{1}{2}\left[\nabla_{i}\left(\nabla_{k} h_{j k}-h_{j k} \nabla_{k} f\right)+\nabla_{j}\left(\nabla_{k} h_{i k}-h_{i k} \nabla_{k} f\right)\right] \\
= & -\frac{1}{2}\left(\nabla_{i} \nabla_{k} h_{j k}+\nabla_{j} \nabla_{k} h_{i k}-\nabla_{k} f \nabla_{i} h_{j k}-\nabla_{k} f \nabla_{j} h_{i k}\right) \\
& -\frac{1}{2}\left(R_{i k} h_{k j}+R_{j k} h_{k i}\right)+\frac{1}{2 \tau} h_{i j} .
\end{aligned}
$$


Now, combining the above computations, we arrive at

$$
\begin{aligned}
\delta R c+\delta \nabla^{2} f= & -\frac{1}{2} \Delta_{f} h-R m(h, \cdot)-\operatorname{div}_{f}^{\dagger} \operatorname{div}_{f} h \\
& -\nabla^{2}\left(-\delta f+\frac{1}{2} \operatorname{tr}_{g} h\right)+\frac{1}{2 \tau} h .
\end{aligned}
$$

Next we derive the variation $\delta \tau$ of the parameter $\tau$.

Lemma 2.4. We have

$$
\delta \tau=\tau \frac{\int_{M}<R c, h>e^{-f}}{\int_{M} R e^{-f}} .
$$

Proof. First of all, from (1.1) we get

$$
R+\Delta f=\frac{n}{2 \tau} .
$$

Also, it is well-known that

$$
R+|\nabla f|^{2}=\frac{f-\nu}{\tau}
$$

From (2.6) and (2.7) it follows that

$$
-\Delta_{f} f=:|\nabla f|^{2}-\Delta f=\frac{f-\nu-n / 2}{\tau} .
$$

Moreover, from (2.4) and (2.5) and using (1.1), we get

$$
\delta R=-\frac{1}{2 \tau} \operatorname{tr}_{g} h+h_{i j} \nabla_{i} \nabla_{j} f+\nabla_{i} \nabla_{j} h_{i j}-\Delta \operatorname{tr}_{g} h,
$$

and

$$
\delta(\Delta f)=\Delta(\delta f)-h_{i j} \nabla_{i} \nabla_{j} f-\nabla_{i} h_{i j} \nabla_{j} f+\frac{1}{2} \nabla_{i} \operatorname{tr}_{g} h \nabla_{i} f
$$

respectively. Also,

$$
\delta|\nabla f|^{2}=2 \nabla_{i} f \nabla_{j}(\delta f)-h_{i j} \nabla_{i} f \nabla_{j} f .
$$

When we integrate (2.2) against the measure $(4 \pi \tau)^{-\frac{n}{2}} e^{-f} d V$ and use (2.3), we obtain

$$
(4 \pi \tau)^{-\frac{n}{2}} \int_{M} \tau\left(|\nabla f|^{2}+R\right) e^{-f} \mathrm{~d} V=\frac{n}{2} .
$$

On the other hand, by differentiating (2.1) and (2.3), we have

$$
(4 \pi \tau)^{-\frac{n}{2}} \int_{M}\left(-\frac{n}{2 \tau} \delta \tau-\delta f+\frac{1}{2} \operatorname{tr}_{g} h\right) e^{-f}=0
$$

and

$$
(4 \pi \tau)^{-\frac{n}{2}} \int_{M} f\left(-\frac{n}{2 \tau} \delta \tau-\delta f+\frac{1}{2} \operatorname{tr}_{g} h\right) e^{-f}+(4 \pi \tau)^{-\frac{n}{2}} \int_{M} \delta f e^{-f}=0 .
$$

Now, differentiating (2.2) and using (2.6), (2.10) and (2.11), we obtain

$$
\begin{aligned}
& 0=\delta \tau\left(-\frac{n}{2 \tau}+|\nabla f|^{2}-\Delta f\right)-\delta f \\
& +\tau\left(-2 \Delta(\delta f)+2 h_{i j} \nabla_{i} \nabla_{j} f+2 \nabla_{i} h_{i j} \nabla_{j} f-\nabla_{i}\left(\operatorname{tr}_{g} h\right) \nabla_{i} f\right. \\
& \left.+2 \nabla_{i} f \nabla_{i}(\delta f)-h_{i j} \nabla_{i} f \nabla_{j} f-\delta R\right) \text {. }
\end{aligned}
$$


Substituting (1.1) and (2.9) in the above identity, we get

$$
\begin{aligned}
0= & -\frac{n}{2 \tau} \delta \tau-2 \tau \Delta(\delta f)+2 \tau \nabla(\delta f) \nabla f-\delta f+\delta \tau\left(|\nabla f|^{2}-\Delta f\right) \\
& +\tau\left(2 h_{i j} \nabla_{i} \nabla_{j} f+2 \nabla_{i} h_{i j} \nabla_{j} f-\nabla_{i}\left(\operatorname{tr}_{g} h\right) \nabla_{i} f-h_{i j} \nabla_{i} f \nabla_{j} f\right) \\
& +\tau\left(\frac{1}{2 \tau} \operatorname{tr}_{g} h-h_{i j} \nabla_{i} \nabla_{j} f-\nabla_{i} \nabla_{j} h_{i j}+\Delta \operatorname{tr}_{g} h\right) .
\end{aligned}
$$

But, by definition of $\operatorname{div}_{f}$, we compute that

$$
\begin{aligned}
\operatorname{div}_{f} \operatorname{div}_{f} h & =\nabla_{i}\left(\nabla_{j} h_{i j}-h_{i j} \nabla_{j} f\right)-\nabla_{i} f\left(\nabla_{j} h_{i j}-h_{i j} \nabla_{j} f\right) \\
& =\nabla_{i} \nabla_{j} h_{i j}-h_{i j} \nabla_{i} \nabla_{j} f-2 \nabla_{i} f \nabla_{j} h_{i j}+h_{i j} \nabla_{i} f \nabla_{j} f .
\end{aligned}
$$

Hence, we get

$$
0=\left(-\frac{n \delta \tau}{2 \tau}-\delta f+\frac{1}{2} \operatorname{tr}_{g} h\right)+\delta \tau\left(-\Delta_{f} f\right)+\tau \Delta_{f}\left(-2 \delta f+\operatorname{tr}_{g} h\right)-\tau \operatorname{div}_{f} \operatorname{div}_{f} h .
$$

Multiplying the above identity by $f$ and integrating against the measure $(4 \pi \tau)^{-\frac{n}{2}} e^{-f} d V$, we get

$$
\begin{aligned}
0= & (4 \pi \tau)^{-\frac{n}{2}} \int_{M} f\left(-\frac{n}{2 \tau} \delta \tau-\delta f+\frac{1}{2} \operatorname{tr}_{g} h\right) e^{-f} d V \\
& +(4 \pi \tau)^{-\frac{n}{2}} \delta \tau \int_{M} f\left(-\Delta_{f} f\right) e^{-f} \mathrm{~d} V \\
& +(4 \pi \tau)^{-\frac{n}{2}} \int_{M} \tau f \Delta_{f}\left(-2 \delta f+\operatorname{tr}_{g} h\right) e^{-f} d V \\
& -(4 \pi \tau)^{-\frac{n}{2}} \int_{M} \tau f\left(\operatorname{div}_{f} \operatorname{div}_{f} h\right) e^{-f} d V
\end{aligned}
$$

By (2.14) and integration by parts, the above identity becomes

$$
\begin{aligned}
0= & (4 \pi \tau)^{-\frac{n}{2}} \int_{M}-\delta f e^{-f} \mathrm{~d} V+\delta \tau(4 \pi \tau)^{-\frac{n}{2}} \int_{M}|\nabla f|^{2} e^{-f} \mathrm{~d} V \\
& +(4 \pi \tau)^{-\frac{n}{2}} \int_{M} \tau\left(-2 \delta f+\operatorname{tr}_{g} h\right) \Delta_{f} f e^{-f} \mathrm{~d} V \\
& -(4 \pi \tau)^{-\frac{n}{2}} \int_{M} \tau<h, \nabla^{2} f>e^{-f} \mathrm{~d} V
\end{aligned}
$$

Using (1.1), (2.8) and (2.12), we obtain

$$
\begin{aligned}
0= & -(4 \pi \tau)^{-\frac{n}{2}} \int_{M} \delta f e^{-f} \mathrm{~d} V+\frac{n}{2 \tau} \delta \tau-\delta \tau(4 \pi \tau)^{-\frac{n}{2}} \int_{M} R e^{-f} \mathrm{~d} V \\
& +(4 \pi \tau)^{-\frac{n}{2}} \int_{M} 2 \tau\left(\frac{n}{2 \tau} \delta \tau+\delta f-\frac{1}{2} \operatorname{tr}_{g} h\right)\left(\frac{1}{\tau} f-\frac{\nu}{\tau}-\frac{n}{2 \tau}\right) e^{-f} \mathrm{~d} V \\
& +(4 \pi \tau)^{-\frac{n}{2}} \int_{M}\left(-\frac{1}{2} \operatorname{tr}_{g} h+\tau h_{i j} R_{i j}\right) e^{-f} \mathrm{~d} V .
\end{aligned}
$$

By using (2.13) and (2.14), we arrive at 


$$
\begin{aligned}
0= & (4 \pi \tau)^{-\frac{n}{2}} \int_{M}\left(\frac{n}{2 \tau} \delta \tau+\delta f-\frac{1}{2} \operatorname{tr}_{g} h\right) e^{-f} \mathrm{~d} V-\delta \tau(4 \pi \tau)^{-\frac{n}{2}} \int_{M} R e^{-f} \mathrm{~d} V \\
& +(4 \pi \tau)^{-\frac{n}{2}} \int_{M} \tau R_{i j} h_{i j} e^{-f} \mathrm{~d} V \\
= & -\delta \tau(4 \pi \tau)^{-\frac{n}{2}} \int_{M} R e^{-f} \mathrm{~d} V+(4 \pi \tau)^{-\frac{n}{2}} \int_{M} \tau R_{i j} h_{i j} e^{-f} \mathrm{~d} V .
\end{aligned}
$$

Therefore,

$$
\delta \tau=\tau \frac{\int_{M} R_{i j} h_{i j} e^{-f} \mathrm{~d} V}{\int_{M} R e^{-f}} .
$$

Now, by Lemmas 2.3 and 2.4 , the second variation becomes

$$
\begin{aligned}
\delta^{2} \nu_{g}(h, h)= & (4 \pi \tau)^{-\frac{n}{2}} \int_{M}-\tau<h, \delta R c+\delta \nabla^{2} f-\frac{1}{2 \tau} h>e^{-f} \\
& +(4 \pi \tau)^{-\frac{n}{2}}\left(-\frac{\delta \tau}{2 \tau}\right) \int_{M} \operatorname{tr}_{g} h e^{-f} \\
= & (4 \pi \tau)^{-\frac{n}{2}} \int_{M} \tau<h, \frac{1}{2} \Delta_{f} h+R m(h, \cdot)+\operatorname{div}_{f}^{\dagger} \operatorname{div}_{f} h>e^{-f} \\
& +(4 \pi \tau)^{-\frac{n}{2}} \int_{M} \tau<h, \nabla^{2}\left(-\delta f+\frac{1}{2} \operatorname{tr}_{g} h\right)>e^{-f} \\
& +(4 \pi \tau)^{-\frac{n}{2}}\left(-\frac{\delta \tau}{2 \tau}\right) \int_{M} \operatorname{tr}_{g} h e^{-f} \\
= & \tau(4 \pi \tau)^{-\frac{n}{2}} \int_{M}<h, \frac{1}{2} \Delta_{f} h+R m(h, \cdot)+\operatorname{div}_{f}^{\dagger} \operatorname{div}_{f} h+\frac{1}{2} \nabla^{2} \hat{v}_{h}>e^{-f} \\
& +\tau(4 \pi \tau)^{-\frac{n}{2}} \frac{\delta \tau}{\tau} \int_{M}<h, \nabla^{2} f-\frac{1}{2 \tau} g>e^{-f} \\
= & \tau(4 \pi \tau)^{-\frac{n}{2}} \int_{M}<h, \frac{1}{2} \Delta_{f} h+R m(h, \cdot)+\operatorname{div}_{f}^{\dagger} \operatorname{div}_{f} h+\frac{1}{2} \nabla^{2} \hat{v}_{h}>e^{-f} \\
& -\tau(4 \pi \tau)^{-\frac{n}{2}} \frac{\int_{M}<R c, h>e^{-f} d V}{\int_{M} R e^{-f} d V} \int_{M}<h, R c>e^{-f} d V .
\end{aligned}
$$

Here,

$$
\hat{v}_{h}=-2 \delta f+\operatorname{tr}_{g} h-\frac{2 \delta \tau}{\tau}(f-\nu)
$$

and it is straightforward to check that

$$
\Delta_{f} \hat{v}_{h}+\frac{\hat{v}_{h}}{2 \tau}=\operatorname{div}_{f} \operatorname{div}_{f} h, \quad \int_{M} \hat{v}_{h} e^{-f} d V=0 .
$$

To see the uniqueness of the solution to (2.15), it suffices to show that $\lambda_{1}\left(\Delta_{f}\right)>$ $\frac{1}{2 \tau}$, where $\lambda_{1}=\lambda_{1}\left(\Delta_{f}\right)$ denotes the first eigenvalue of $\Delta_{f}$. Let $u$ be a (non-constant) first eigenfunction so that

$$
\Delta_{f} u=-\lambda_{1} u
$$


Then by direct computations (see also [9]), we get

$$
\begin{aligned}
\frac{1}{2} \Delta_{f}|\nabla u|^{2} & =\left|\nabla^{2} u\right|^{2}+\nabla\left(\Delta_{f} u\right) \cdot \nabla u+\left(R c+\nabla^{2} f\right)(\nabla u, \nabla u) \\
& \geq \frac{1}{n}|\Delta u|^{2}+\left(\frac{1}{2 \tau}-\lambda_{1}\right)|\nabla u|^{2} .
\end{aligned}
$$

Thus,

$$
0=\int_{M} \frac{1}{2} \Delta_{f}|\nabla u|^{2} e^{-f} d V \geq \frac{1}{n} \int_{M}|\Delta u|^{2} e^{-f} d V+\left(\frac{1}{2 \tau}-\lambda_{1}\right) \int_{M}|\nabla u|^{2} e^{-f} d V .
$$

Since $u$ is non-constant, we obtain

$$
\lambda_{1}>\frac{1}{2 \tau} .
$$

This completes the proof of Theorem 1.1 .

\section{Further Remarks And the proof of Theorem 1.3}

Recall that a gradient shrinking Ricci soliton $\left(M^{n}, g_{i j}, f\right)$ is called linearly stable if the stability operator $\hat{N} \leq 0$ on symmetric 2-tensors. Note that $\hat{N}$ is degenerate negative elliptic. In this section we shall exhibit the action of the stability operator on a couple of special symmetric 2 -tensors $h$ : (i) $h_{i j}=g_{i j}$ and (ii) $h_{i j}=R_{i j}$, and prove Theorem 1.3.

Without loss of generality, we assume $\tau=1$ so that our shrinking soliton $\left(M^{n}, g_{i j}, f\right)$ satisfies the equation

$$
R_{i j}+\nabla_{i} \nabla_{j} f=\frac{1}{2} g_{i j}
$$

We also normalize $f$ so that

$$
(4 \pi)^{-\frac{n}{2}} \int_{M} e^{-f}=1
$$

First of all, notice that we have

$$
\begin{gathered}
\Delta_{f} g=\Delta g-\nabla f \nabla g=0 \\
\operatorname{div}_{f} g=-g_{i j} \nabla_{j} f=-\nabla f, \quad \operatorname{div}_{f}^{\dagger} \operatorname{div}_{f} g=\nabla^{2} f \\
\hat{v}_{h}=-2(f-\bar{f}), \quad \bar{f}=(4 \pi \tau)^{-\frac{n}{2}} \int_{M} f e^{-f}
\end{gathered}
$$

Hence, we get

$$
\hat{N}(g)=R c+\nabla^{2} f+\frac{1}{2} \nabla^{2}(-2 f+2 \bar{f})-R c=0
$$

as we expected.

On the other hand, we have

Lemma 3.1. For any complete shrinking Ricci soliton satisfying (3.1), we have

$$
R c \in \operatorname{ker} \operatorname{div}_{f} .
$$


Proof. By definition and the second contracted Bianchi identity,

$$
\left(\operatorname{div}_{f} R c\right)_{i}=\nabla_{j} R_{i j}-R_{i j} \nabla_{j} f=\frac{1}{2} \nabla_{i} R-R_{i j} \nabla_{j} f .
$$

On the other hand, it is a basic fact that our shrinker satisfies

$$
\nabla_{i} R=2 R_{i j} \nabla_{j} f
$$

Therefore, $\operatorname{div}_{f}(R c)=0$.

Recall the operator $\mathcal{L}_{f}$ on symmetric 2 -tensors defined in (1.10):

$$
\mathcal{L}_{f} h:=\frac{1}{2} \Delta_{f} h+\operatorname{Rm}(h, \cdot) .
$$

It is easy to see that $\mathcal{L}_{f}$ is a self-adjoint operator with respect to the weighted $L^{2}$-inner product $(\cdot, \cdot)_{f}$ defined in $(1.6)$.

Lemma 3.2. For any complete shrinking soliton satisfying (3.1), its Ricci tensor is an eigen-tensor of the operator $\mathcal{L}_{f}$ with eigenvalue $-1 / 2$ :

$$
\mathcal{L}_{f}(R c)=\frac{1}{2} R c
$$

Proof. The following computations are familiar to experts, but we carry out here for completeness.

From the soliton equation (3.1), we have

$$
R_{i j}=\frac{1}{2} g_{i j}-\nabla_{i} \nabla_{j} f
$$

By commuting covariant derivatives, we have

$$
\begin{aligned}
\Delta R_{i j} & =-\nabla_{k} \nabla_{k} \nabla_{i} \nabla_{j} f \\
& =-\nabla_{k}\left(\nabla_{i} \nabla_{k} \nabla_{j} f+R_{k i j l} \nabla_{l} f\right) \\
& =-\nabla_{k} \nabla_{i} \nabla_{j} \nabla_{k} f-\nabla_{k} R_{k i j l} \nabla_{l} f-R_{k i j l} \nabla_{k} \nabla_{l} f .
\end{aligned}
$$

On the other hand, by commuting covariant derivatives again and using the contracted second Bianchi identity as well as (3.1), we obtain

$$
\begin{aligned}
\nabla_{k} \nabla_{i} \nabla_{j} \nabla_{k} f & =\nabla_{i} \nabla_{k} \nabla_{j} \nabla_{k} f+R_{k i j l} \nabla_{l} \nabla_{k} f+R_{k i k l} \nabla_{j} \nabla_{l} f \\
& =-\frac{1}{2} \nabla_{i} \nabla_{j} R-R_{k i j l} R_{k l}-R_{i l} R_{l j} \\
& =-\nabla_{j} R_{i l} \nabla_{l} f-\frac{1}{2} R_{i j}-R_{k i j l} R_{k l}
\end{aligned}
$$

Here we have used (3.3) in deriving the last equality.

Moreover, by the second Bianchi identity, we have

$$
\nabla_{k} R_{k i j l} \nabla_{l} f=\left(\nabla_{j} R_{i l}-\nabla_{l} R_{i j}\right) \nabla_{l} f .
$$

Combining the above calculations and using the Ricci soliton equation (3.1), we arrive at

$$
\Delta R_{i j}=\nabla_{l} R_{i j} \nabla_{l} f+2 R_{k i j l} R_{k l}+R_{i j},
$$

i.e., $2 \mathcal{L}_{f}\left(R_{i j}\right)=R_{i j}$. 
Now, for any $h \in \operatorname{ker}_{\operatorname{div}}$, the stability operator $\hat{N}$ is given by

$$
\hat{N} h=\mathcal{L}_{f} h-R c \frac{\int_{M}<R c, h>e^{-f}}{\int_{M} R e^{-f}} .
$$

Moreover, from (3.4) we obtain

$$
\Delta_{f} R=R-2|R c|^{2},
$$

from which it follows that

$$
2 \int_{M}|R c|^{2} e^{-f}=\int_{M} R e^{-f} .
$$

Therefore, by Lemma 3.2, (3.5) and (3.7), we have

\section{Lemma 3.3.}

$$
\hat{N}(R c)=0 .
$$

Now we are ready to prove

Proposition 3.1. Suppose $\left(M^{n}, g_{i j}, f\right)$ is a linearly stable compact shrinking soliton satisfying (3.1), then $-1 / 2$ is the only negative eigenvalue of the operator $\mathcal{L}_{f}$ on $\operatorname{ker} \operatorname{div}_{f}$, and the multiplicity of $-1 / 2$ is one. In particular, $-1 / 2$ is the least eigenvalue of $\mathcal{L}_{f}$ on $\operatorname{ker} \operatorname{div}_{f}$.

Proof. By Lemma 3.1 and Lemma 3.2, we know that $R c \in \operatorname{ker} \operatorname{div}_{f}$, and is an eigentensor of $\mathcal{L}_{f}$ with eigenvalue $-1 / 2$. Suppose there exists a (non-zero) symmetric 2 -tensor $h \in \operatorname{ker}_{\operatorname{div}}$ such that

$$
\mathcal{L}_{f} h=\alpha h,
$$

with $\alpha>0$, and

$$
(R c, h)_{f}=: \int_{M}<R c, h>e^{-f}=0 .
$$

Then, by Theorem 1.1 and (3.5), we have

$$
\begin{aligned}
\delta^{2} \nu_{g}(h, h) & =\frac{1}{(4 \pi)^{n / 2}} \int_{M}<\hat{N} h, h>e^{-f} \\
& =\frac{1}{(4 \pi)^{n / 2}} \int_{M}<\mathcal{L}_{f} h, h>e^{-f} \\
& =\frac{\alpha}{(4 \pi)^{n / 2}} \int_{M}|h|^{2} e^{-f}>0,
\end{aligned}
$$

a contradiction to the linear stability of $\left(M^{n}, g_{i j}, f\right)$. Thus $-1 / 2$ is the only negative eigenvalue of $\mathcal{L}_{f}$ on $\operatorname{ker}_{\operatorname{div}_{f}}$, with multiplicity one.

Remark 3.1. In [10, the authors have given a very nice interpretation of their proof in terms of the multiplicity of the eigenvalue $-1 / 2$ : for any compact shrinking Kähler-Ricci soliton satisfying (3.1), the eigen-space of eigenvalue $-1 / 2$ has multiplicity at least $h^{1,1}$. Hence a compact shrinking Kähler-Ricci soliton with $h^{1,1}>1$ is unstable. 


\section{REFERENCES}

[1] Cao, H.-D., Existence of gradient Kähler-Ricci solitons, Elliptic and Parabolic Methods in Geometry (Minneapolis, MN, 1994), 1-16, A K Peters, Wellesley, MA 1996.

[2] Cao, H.-D., Recent progress on Ricci solitons, Recent advances in geometric analysis, Adv. Lect. Math. (ALM) 11, 1-38, International Press, Somerville, MA 2010.

[3] Cao, H.-D., Geometry of complete shrinking Ricci solitons, Geometry and Analysis (Vol. I), Adv. Lect. Math. (ALM) 17, 227-246, International Press, Somerville, MA 2011.

[4] Cao, H.-D., Hamilton, R. S. and Ilmanen, T., Gaussian densities and stability for some Ricci solitons, arXiv:math.DG/0404165

[5] Cao, H.-D. and Zhou, D., On complete gradient shrinking solitons, J. Differential Geom. 85 (2010), 175-185.

[6] Cao, H.-D. and Zhu, X.-P., A complete proof of the Poincaré and geometrization conjectures - application of the Hamilton-Perelman theory of the Ricci flow, Asian J. Math. 10 (2006), 165-492.

[7] Colding, T. H. and Minicozzi, W. P., Generic mean curvature flow I; generic singularities, arXiv:0908.3788

[8] Feldman, M., Ilmanen, T. and Knopf, D., Rotationally symmetric shrinking and expanding gradient Kähler-Ricci solitons, J. Differential Geom., 65 (2003), 169-209.

[9] Futaki, A. and Sano, Y., Lower diameter bounds for compact shrinking Ricci solitons, arXiv:1007.1759.

[10] Hall, S. and Murphy, T., On the linear stability of Kähler-Ricci solitons, Proc. Amer. Math. Soc. 139 (2011), 3327-3337.

[11] Hamilton, R. S., The Ricci flow on surfaces, Contemporary Mathematics 71 (1988), 237-261.

[12] Ivey, T., Ricci solitons on compact three-manifolds, Diff. Geom. Appl. 3 (1993), 301-307.

[13] Koiso, N., On rotationally symmetric Hamilton's equation for Kähler-Einstein metrics, Recent Topics in Diff. Anal. Geom., Adv. Studies Pure Math., 18-I, 327-337, Academic Press, Boston, MA 1990.

[14] Munteanu, O. and Sesum, N., On gradient solitons, arXiv:0910.1105

[15] Perelman, G., The entropy formula for the Ricci flow and its geometric applications, arXiv:math.DG/0211159

[16] Wang, X.- J. and Zhu, X.- H., Kähler-Ricci solitons on toric manifolds with positive first Chern class, Adv. Math. 188 (2004), 87-103.

[17] Zhu, M., The second variation of the Ricci expanding entropy, Pacific J. Math. 251 (2011), 499-510.

Department of Mathematics, Lehigh University, Bethlehem, PA 18015, USA

E-mail address: huc2@lehigh.edu \& mez206@lehigh.edu 